\title{
Early Experience in Aortic Valve Reconstruction with Autologous Pericardium for Aortic Valve Insufficiency
}

\author{
Magdy Hassanein ${ }^{1 *}$ and Ahmed Zayed ${ }^{2}$ \\ ${ }^{1}$ Department of Cardiac Surgery, National Heart Institute, Cairo, Egypt \\ 2Department of Cardiology, National Heart Institute, Cairo, Egypt
}

Received: 30May, 2017 ; Accepted: 12 July, 2017; Published: 21 July, 2017

*Corresponding author: Magdy Hassanein MD, Department of Cardiac Surgery, National Heart Institute, Cairo, Egypt, E-mail: megs660@yahoo. com

\begin{abstract}
Objective: Several authors have reported good results with the use of autologous treated pericardium for aortic valve reconstruction with good results specially the current series of Ozaki, so we decided to start our learning curve in this type of surgery for its benefits and to avoid the cost and potential complications that may be resulted from aortic valve replacement.
\end{abstract}

Methods: Between August 2013 and November 2015, 43 patients were scheduled for aortic valve reconstruction using gluataraldehyde treated autologous pericardium. They were isolated aortic lesions or associated with repairable mitral or mitral and tricuspid valve lesion (26 patients were pure aortic regurge, 8 patients were aortic regurge + mild to moderate stenosis, 5 patients were aortic valve lesion associated with mitral regurge and 4 patients were aortic lesions associated with mitral and tricuspid regurge). Their mean age was $30.4 \pm 6.7$ and male/female was 25/18. We used Gaspayran formula to fabricate new pericardial leaflet, all patients were evaluated by transesophygeal echo before discharging from the operating room.

Results: 4 patients out of 43 patients have undergone aortic valve replacement ( 2 of them because of mitral valve repair failed and the other 2 because of thickened pericardium and adhesion) aortic valve reconstruction was done successfully in the remaining patients (39 patients).There were no hospital deaths, transthoracic echo before discharge from the hospital revealed no regurge and no significant pressure gradient, also follow up echo for a mean period $21.5 \pm 6.9$ months revealed stable aortic valve function with no significant pressure gradient or significant regurge.

Conclusion: Aortic valve reconstruction with treated autologous pericardium is feasible with acceptable hemodynamic. The cornerstone of this surgery is perfect sizing and fabricating the new pericardial aortic leaflets and precise suturing methods.

Keywords: Aortic valve reconstruction; Autologous pericardium Geometric description of aortic root

\section{Introduction}

Mitral and tricuspid valve repair have become more popular than valve replacement due to their standardization and reproducibility. However, for aortic valve disease, aortic valve replacement is a commonly performed procedure and provides satisfactory long term results [1]. Replacement with mechanical prosthesis in a young patient has drawbacks of long-term anticoagulation and definite risk for thromboembolic complications [2]. Bioprosthesis implantation is the preferred choice for AVR in the elderly as a result of the shorter life expectancy and reduced need of anticoagulation. However, despite the progress of the design and construction of prosthetic valves, hemodynamic performance is not yet comparable to that of native aortic valves [1].

In the 1960s, Bajoerk and Hultquist first implanted autologous pericardial leaflets with a technique similar to that used today with untreated pericardium. However, scarring and retraction were common, and the procedure was abandoned. In 1986, love and colleagues first reported immersing autologous pericardium in $0.6 \%$ glutaraldehyde for 10 minutes and eliminating the scarring problem [3].

In recent years with marked improvement in myocardial protection, much attention has been given to re-examined old surgical techniques that were applied intermittently by isolated surgeons to a small number of patients. Aortic value reconstruction (AoRec) using a single strip of autologous pericardium fixed in glutaraldehyde described by Duran et al. with satisfactory results [4]. Al Halees and Colleagues published a series of glutaraldehyde-fixed autologous pericardium valves in 65 young patients with excellent outcomes. Ozaki and Colleagues current series of 591 patients has shown excellent valve function to a maximal follow up of 6 years [3].

Because of these excellent and satisfactory results of the previous researches and the advantages of the constructed valve (stentless, inexpensive, lacks antigenicity and easily constructed in operating room), we encourage to start our experience is aortic valve reconstruction in selected patients with aortic valve insufficiency either pure regurge or combined with mild to moderate stenosis but without calcification and isolated aortic valve disease or combined with reparable mitral valve disease especially we are in developing country and have some ignorant 
patients who usually lose INR follow up.

\section{Patients and Methods}

Between August 2013 and November 2015, we attempted aortic valve reconstruction with glutaraldehyde treated autologous pericardium in 43 patients. All patients were evaluated by pre-operative transthoracic echocardiography. The clinical data of the patients is represented in table [1]. The indications of surgery were according to the standard published guidelines for symptomatic and asymptomatic patients with valvular heart disease. Each patient signed an informed consent form, and study protocol was approved by the local medical research committee.

\begin{tabular}{|l|c|}
\hline \multicolumn{2}{|l|}{ Table 1: Preoperative data of the patients (n=43) } \\
\hline Variable & $30.4 \pm 6.7$ \\
\hline Age (years) & $25 / 18$ \\
\hline 17 y-47 y & \\
\hline Sex M/F & 32 \\
\hline NYHA functional class & 11 \\
\hline Class II & 0 \\
\hline Class III & \\
\hline Class IV & 34 \\
\hline Preoperative echo & 26 \\
\hline Isolated aortic lesion & 8 \\
\hline Pure aortic regurge & 5 \\
\hline Aortic regurge + mild to moderate stenosis & 4 \\
\hline Combined aortic lesion + mitral valve regurge & $23.6 \pm 1.8$ \\
\hline Aortic lesion + mitral regurge \& tricuspid & $56.7 \pm 3.4$ \\
\hline regurge & \\
\hline Mean peak pressure gradient (mmHg) 11 - 28 & \\
\hline Mean surgical annular diameter (mm) 20 - 26 & \\
\hline Ejection fraction (EF) \% & \\
\hline
\end{tabular}

In this study, we operated upon patients with pure aortic regurge or combined lesion (mild to moderate stenosis) either isolated aortic valve disease or combined with only repairable mitral valve disease or repairable tricuspid valve. Patients with pure aortic stenosis or heavily calcified valve or combined with other cardiac lesion except repairable MV and/or tricuspid valve were excluded from our study. We also excluded bicuspid aortic valve from this study.

All cases were rheumatic, tricuspid leaflets with no or very mild calcification.

\section{Surgical Technique}

Under general anaesthesia (TEE) was performed in all patients to assess the aortic valve, annular diameter, other cardiac valves as well as LV function, then median sternotomy was done, autologous pericardium was excised after cleaning from the loose areolar tissue by blunt dissection, the patch of the pericardium is pinned flat on a sterile cardboard and treated with $0.6 \%$ glutaraldehyde solution for 10 minutes, then washed 3 times in saline ( 3 minutes each time).

Cardiopulmonary bypass was established using ascending aorta and two-stage right atrial cannulation in patients with isolated aortic valve lesion or bicavalcannulation in patients with associated mitral and/or tricuspid valve lesions. Myocardial protection was done using cold blood cardioplegia directly into the coronary ostia.

Unfortunately, we don't have any seizer for this purpose (aortic valve reconstruction), so reviewed most of published anatomical studies about the relationship between intercommissural distance, length of free margin of aortic leaflet, height of leaflet and etc.

Finally, we decided to apply the formula of Gasparyan using a compass, ruler and medical pen to measure and fabricate the new aortic valve leaflets from the autologous pericardium. After a bypass and cross clamp, the aorta was opened transversely about $0.5 \mathrm{~cm}$ above the sinotubular junction and cold blood cardioplegia was taken directly into the coronary ostia.

In cases associated with mitral valve lesion, we attack mitral valve first through conventional left atriotomy and mitral valve repair done using posterior band annuloplasty with or without some additional procedures like artificial chardea (GorTex suture). Only if mitral repaired successfully, aortic valve reconstruction was performed.

Stay sutures were placed through the adventita close to the edge of the lower flap of aortotomy to keep the aortotomy open and good exposure to the aortic valve. Aortic valve leaflets were excised leaving 2 to $3 \mathrm{~mm}$ of the cusp bases [5]. Intercommissural distances were measured using a compass.

Fabricating of new aortic valve leaflets was done from washed fixed autologous pericardium after mathematically calculation of length of the free edge, height and radius of the aortic valve leaflets as well as the commissural height using Gaspayran formula [6] Where,

$$
\begin{array}{ll}
-\mathrm{L}(\mathrm{r}) & =1.2 \times \mathrm{I}(\mathrm{r}) \\
-\mathrm{L}(\mathrm{l}) & =1.2 \times \mathrm{I}(\mathrm{l}) \\
-\mathrm{L}(\mathrm{n}) & =1.2 \times \mathrm{I}(\mathrm{n}) \\
-\mathrm{H} & =0.866 \times \text { mean I } \\
-\mathrm{K} & =0.266 \times \text { mean I } \\
-\mathrm{R} & =0.6 \times \text { mean I }
\end{array}
$$

Where,

-L (n), L (r) and L (l) mean length of the free edge

-L (n), I (r) and I (l)mean inter commissural distances

-H means (height of the leaflet)

-K means (commissural height) 
-R means (radius of leaflet attachment line)

( $\mathrm{n}=$ Non-coronary cusp, $\mathrm{r}=$ right coronary cusp, $\mathrm{l}=$ left coronary cusp)

Finally, we got three new aortic valve leaflets nearly identical and attached to each other, we mark the center of the fabricated aortic valve leaflets using medical pen. The pericardial leaflets were oriented so that the smooth forces of all leaflets faced the coaptation surface (ventricular side).

Three 4/0 double-arm prolene sutures through the mid-point of each pericardial leaflets and the center of the corresponding sinus, additional two $4 / 0$ prolene used to hold the commissures in place with the aortic wall and kept untied during sewing of the new leaflets with corresponding sinuses.

We use Ozaki et al. methods for suturing technique; the suture is begun at the center of the annulus. This suture is tied and the ends are continued as a running suture bidirectionally. The ratio of distance between each bite on the pericardium and annulus 3:1 at the bottom of the annulus and then changed to 1:1 when the suture comes up to the coaptation zone.

Once this running suture has reached the commissure, it is brought out through the aortic wall and kept until the running suture of another cusp also reached the commissure and brought out through the aortic wall, these two sutures were tied to each other over a pledget. A similar procedure is done for the other sutures.

The coaptation of the 3 new leaflets was ensured with direct vision, under negative pressure made by a left ventricular vent before closure of aortotomy.

\section{Results}

During the period of study 43 patients were operated upon for aortic valve reconstruction by washed fixed autologous pericardium. They were 34 patients isolated aortic lesion (26 patients pure aortic regurge and 8 patients were regurge+ mild to moderate aortic stenosis). 9 patients were aortic valve lesion associated with mitral regurge or mitral and tricuspid regurge; 4 patients out of 43 patients have undergone prosthetic aortic valve replacement ( 2 of them because of failure of mitral valve repair associated with the aortic valve lesion\& the other 2 because of adhesion of the pericardium to the heart although it was a soft adhesion but the pericardium was thick.

So, 39 patients have undergone aortic valve reconstruction (32 patients $\mathrm{AV}$ reconstruction only and 7 patients AV reconstruction \& mitral or mitral and tricuspid valve repair with flexible band).

Transesophageal echocardiography (TEE) in operating room postoperative revealed satisfactory result in all patients except one patient in which moderate to severe eccentric mitral valve regurge, we resumed cardiopulmonary bypass in this patient and artificial chordae was added to the mitral valve repair and TEE postoperative showed competent mitral valve repair.

Mean aortic cross clamp time was $95 \pm 22.7$ minutes in isolated aortic valve reconstruction and $122.4 \pm 26.9$ in patients with double valve repair. Mean cardiopulmonary bypass (CPB) time was $115.7 \pm 23.4$ and $149.6 \pm 29.3$ minutes with isolated aortic valve reconstruction and double valve repair respectively. There were no hospital deaths.

\section{Follow-up}

Transthoracic echocardiography was performed prior to discharge and was repeated every 3 months for two years. 5 patients lost from follow up after the first 6 months. Aortic valve function remained stable in all patients apart from (2 patients showed trivial aortic regurge) as well as no significant pressure gradient across the aortic valve in all patients during the follow up period which range from 6-24 months, mean $21.5 \pm 6.9$ months.

\section{Discussion}

Actually I took a long time thinking about this type of surgery (aortic valve reconstruction using autologous pericardium) especially we don't' have any device to size and fabricates the new aortic valve leaflets. We tried to get Ozaki seizer and template but it was very expensive to us $(7.000$ dollars). On the other hand many factors are pushing me to try like cost (cost of prosthetic or bioprosthetic valve, cost of long term anticoagulation, repeated monitoring of INR, and repeated visits to the hospital (our economy is not in a good state). Some ignorant people don't care about the INR and anticoagulation. Limitation of the life style of people receiving anticoagulation and complications of thrombo-embolism, the development of TAVI technique and the possibility of valve in valve implantation in the future if the patient needs this as well as the younger patients who received prosthetic aortic valve during they have small surface area and after they grow and have large body surface area patients/prosthesis mismatch will happen and need repoperation. All of the previously mentioned factors pushed us to try aortic valve reconstruction using autologous pericardium. But before we apply it on our patients, we did it many times in our wet lab. Aortic valve reconstruction with autologous pericardium is not new, attempts to replace aortic valve cusp tissue with biologic material have been made since the late 1960s [4].

Fascia lata, dura mater and bovine pericardium have been used in a small number of patients but the results have not been favorable in the majority of instances [7]. Autologous pericardial leaflets replacement has a long history, performed first by Bjork in the early 1960s. Untreated pericardium, however, experienced scarring and retraction and the method subsequently was abandoned. Dr. Jack Love who first immersed autologous pericardium in gluteraldehyde for 10 minutes and this eliminated the scarring problem [8].

Techniques for aortic valve reconstruction have improved over the last 15 years. Several authors have reported good results with the use of autologous treated pericardium for aortic valve reconstruction with good results. Chan and Colleagues reported 11 patients of truly stent less autologous pericardial aortic valve replacements with their 6.5 years of follow-up [7]. Freedom from structural valve deterioration was 
$100 \%$.The important current series is that of Ozaki in Tokyo with minimal valve related complications and excellent autologous valve function in over 400 patients followed to over 5 years and because the dimensions and geometric relationships of the human aortic valve have been well-investigated. We encourage to start this study [7].

Our patients were selected carefully (like a super selection) \& their mean age was somewhat younger than other series we reviewed. So, I think this is the cause of good results in our series. However, the cardiopulmonary bypass time was still long because of the need to fabricate the cusp as well as long suture lines. In conclusion, aortic valve reconstruction with glutaraldehyde treated autologous pericardium is feasible with acceptable hemodynamic results.

\section{References}

1. Ozaki S, Kawase I, Yamashita H, Uchida S, Nozawa Y, Matsuyama T, et al. Aortic valve reconstruction using self-developed aortic valve plasty system in aortic valve disease. Interact CardiovascThorac Surg. 2011;12(4):550-553. doi: 10.1510/icvts.2010.253682

2. Mittal CM, Talwar S, Devagourou V, Kothari SS, Kumar AS. Early results of aortic valve reconstruction with stent less glutaraldehyde treated autologous pericardial valve. Indian J Thorac Cardiovasc Surg. 2009;25(4):178-182.
3. Rankin JS, Nobauer C, Crooke PS, Schreiber C, Lange R, Mazzitelli D, et al. Techniques of autologous pericardial leaflet replacement for aortic valve reconstruction. Ann Thorac Surg. 2014;98(2):743-745. doi: 10.1016/j.athoracsur

4. Duran CM, Gallo R, Kumar N. Aortic valve replacement with autologous pericardium: surgical technique. J Card Surg. 1995;10(1):1-9.

5. Song MG, Yang HS, Choi JB, Shin JK, Chee HK, Kim JS. Aortic valve reconstruction with use of pericardial leaflets in adults with bicuspid aortic valve disease: early and midterm outcomes. Tex Heart Inst J. 2014;41(6):585-591. doi: 10.14503/THIJ-13-3619

6. Gasparyan VC. Reconstruction of the aortic valve with autologous pericardium: an experimental study. J Thorac Cardiovasc Surg. 1999;117(1):197-198.

7. Ozaki S, Kawase I, Yamashita H, Uchida S, Nozawa Y, Takatoh M, et al. A total of 404 cases of aortic valve reconstruction with glutaraldehydetreated autologous pericardium. J Thorac Cardiovasc Surg. 2014;147(1):301-306. doi: 10.1016/j.jtcvs.2012.11.012

8. Rankin JS, Crooke PS, Lange R, Mazzitelli D. Techniques of autologous pericardial leaflet replacement for bicuspid aortic valve endocarditis. J Heart Valve Dis. 2013;22(5):724-731. 